\title{
Reducing False Alarm Rate in Anomaly Detection with Layered Filtering
}

\author{
Rafał Pokrywka ${ }^{1,2}$ \\ ${ }^{1}$ Institute of Computer Science \\ AGH University of Science and Technology \\ al. Mickiewicza 30, 30-059 Kraków, Poland \\ pokrywka@agh.edu.pl \\ ${ }^{2}$ IBM SWG Laboratory ${ }^{\star}$ \\ ul. Armii Krajowej 18, 30-150 Kraków, Poland
}

\begin{abstract}
There is a general class of methods of detecting anomalies in a computer system which are based on heuristics or artificial intelligence techniques. These methods are to distinguish between normal and anomalous system behaviour. The main weakness of these methods is a false alarm rate which is usually measured by counting false-positives on a sample set representing normal behaviour. In this measurement a base rate of anomalous behaviour in a live environment is not taken into account and that leads to a base-rate fallacy. This problem can greatly affect a real number of false alarms which can be significantly greater then expected value. Usually little can be done to further improve classification algorithms. In this paper a different approach to reducing real false alarm rate based on layered filtering is presented and discussed. The solution explores potential in a properly structured system of several anomaly detectors.
\end{abstract}

\section{Introduction}

Work presented here is a part of research on Intrusion Detection System (IDS) which is based on anomaly detection. This kind of security systems are usually placed in key nodes of network infrastructure and are often used to complement signature based detection systems. The system detects anomalies by comparing normal behaviour, stored in some way as a profile, and current behaviour of supervised processes. This approach allows to detect an attack without a priori knowledge of attack's technique in opposite to signature-based detectors which are basically blind to novel attack patterns.

Anomaly detectors sometimes fail to correctly classify current event. From all misclassification types the false-positive error, also referred as false alarm which is when normal behaviour is marked as anomalous, is the most significant. This article focuses on a very important aspect of my research - proper handling of this kind of errors. The goal is to have an effective system yet with very

\footnotetext{
* This paper is NOT related to any of my job responsibilities as an employee of IBM.
} 
low real false alarm rate - even close to 0 . The problem is that real life system usually works fine most of the time and only sometimes abnormal event happens - simply the frequency of anomalies is fairly low. The false alarm rate of anomaly detectors is usually measured by taking the ratio between a number of alarms fired on a sample set of normal events and the size of this set - the base rate is not taken into account in this measurement. The result is that real false alarm rate achieved during monitoring of real systems can be significantly larger then expected - even large enough to make anomaly detector basically useless. This phenomenon is known as base-rate fallacy and directly stems out from Bayes theorem. It also frequently skips the attention of researchers. It is a hard task to further improve current anomaly detection algorithms, which have already achieved quite low false alarm rates, but even large improvement may not be enough. The real improvement can be achieved by combining several anomaly detectors with different properties and performance into layers which gradually filters out abnormal events.

In this article the term "event" is used to describe smallest set of information from the system that can be classified. The term "behaviour" relates to a sequence of events.

\section{Anomaly Detectors Overview}

Anomaly detection algorithms are used to distinguish if a current event is normal or not. To simplify discussion at this moment it can be assumed that information about type of anomaly, like for example error condition or buffer overflow, can be neglected. In this case detector can be seen as binary classifier as there are only two possible classes of events - normal event or anomaly.

There are four possible outcomes from a detector with respect to the actual class of an event:

- true positive (TP) - when current event is anomalous and detector prediction is correct (signalisation of anomaly)

- false positive (FP) - when current event is normal but detector prediction is incorrect (signalisation of anomaly)

- true negative $(\mathrm{TN})$ - when current event is normal and detector prediction is correct (no signalisation of anomaly)

- false negative (FN) - when current event is anomalous but detector prediction is incorrect (no signalisation of anomaly)

The false positive is also known in statistics as type I error and this is the situation when a false alarm is fired. False negative is know as type II error and it describes the situation when real anomaly is missed by the detector. True positive is when a detector correctly signals anomaly and true negative when it correctly indicates normal event.

Anomaly detectors in scientific publications are usually characterized by the following operational characteristics: 
- detection rate (DR) - which is exactly the same as true positive rate and can be calculated using the following equation: $D R=\frac{T P}{T P+F N}$

- false alarm rate (FAR) - which is the same as false positive rate and is calculated using: $F A R=\frac{F P}{F P+T N}$

A good graphical presentation of these two characteristics (DR and FAR) is provided by ROC curve. It allows to conveniently compare different detection algorithms or choose the best set of algorithm parameters. A great survey of a couple of the most popular detection techniques can be found in [3].

\section{The Base-Rate Fallacy}

The difficulty in improving effectiveness of anomaly detectors due to base-rate fallacy phenomenon has been first pointed out by Stefan Axelsson in [2]. The fallacy stems out directly from Bayes theorem which relates prior and posterior probability of an event and is given by the following formula:

$$
P(A \mid B)=\frac{P(A) * P(B \mid A)}{P(B)} .
$$

$P(B)$ can be expressed, using the law of total probability for $n$ mutually exclusive outcomes of $A$, in the following way:

$$
P(B)=\sum_{i=1}^{n} P\left(A_{i}\right) * P\left(B \mid A_{i}\right) .
$$

Finally after combination of (11) and (2) the most popular Bayes theorem form can be derived:

$$
P(A \mid B)=\frac{P(A) * P(B \mid A)}{\sum_{i=1}^{n} P\left(A_{i} * P\left(B \mid A_{i}\right)\right.} .
$$

Following Axellsson let's assume that $I$ means an anomalous event in a system, $\neg I$ that it is a normal event (no anomaly), $A$ that there is an alarm signalisation from a detector and $\neg A$ that there is no alarm fired. The false alarm rate can be expressed by the probability $P(A \mid \neg I)$ and detection rate by $P(A \mid I)$. True negative and false negative rates can be obtained, respectively, in the following way: $P(\neg A \mid \neg I)=1-P(A \mid \neg I)$ and $P(\neg A \mid I)=1-P(A \mid I)$. Equation (3) can now be rewritten as:

$$
P(I \mid A)=\frac{P(I) * P(A \mid I)}{P(I) * P(A \mid I)+P(\neg I) * P(A \mid \neg I)} .
$$

The goal in anomaly detection is to maximise $P(I \mid A)$, which is called by Axellson a Bayesian Detection Rate (BDR), and $P(\neg I \mid \neg A)$, which is the probability that lack of alarm really means lack of anomaly and in this paper will be called Bayesian True Negative Rate (BTNR). In a real life environment the frequency of anomalies is fairly low. Based on [2] the assumptions has been made that 
the average is $2 * 10$ anomalous events per day and $10^{6}$ events overall per day. This allows to calculate the following probabilities: $P(I)=2 * 10^{-5}$ and $P(\neg I)=$ $1-P(I)=0.99998$. Another assumptions has been made about characteristics of hypothetical anomaly detector: $D T=P(A \mid I)=1$ and $F A R=P(A \mid \neg I)=10^{-5}$. In fact such values would be a really great achievement - they are simply not realistic and are only to show how significant base-rate fallacy is. Taking all these values the calculated $B D R$ is 0.66667 . It means that the probability a fired alarm is not a false alarm is only 0.66667 . This value in practice can not be tolerated - it makes anomaly detector useless for an administrator. Under the same base rate assumptions the probability $B T N R=P(\neg I \mid \neg A)=\frac{P(\neg I) * P(\neg A \mid \neg I)}{P(\neg I) * P(\neg A \mid \neg I)+P(I) * P(\neg A \mid I)}$ is dominated by the base rate of normal events and is always close to 1 which means that anomaly rarely escapes detection.

Axelsson argues that it is crucial to keep false alarm rate as low as possible even if the algorithm complexity and resource consumption is very high. This of course makes the detection very slow and there is a risk that intrusion is not detected on time. The potential damages and losses may have already been done. Also further improvement of FAR of current detection algorithms may not be a feasible task - there is to much effort for little gains. Layered Filtering may be an answer for these difficulties.

\section{$4 \quad$ Layered Filtering}

Layered filtering is well known in air or water pollution elimination. It consist of at least two filters in a sequence and each filter is responsible for elimination of different pollution type. The air, for example, flows through all filters and every filter is responsible for eliminating different chemical pollution or dust. As a result a clean air is supposed to be achieved.

Returning back to the computer science field there is a method of combining binary classifiers to get a multiple classifier. This classifier uses more then one specialised binary classifier for each class and combines theirs outcomes - see for example 4 .

The idea behind layered filtering takes something from both analogies: computer science and non-computer science. It gradually filters out normal system events as air filters do with pollution. It is also similar to multiple classifier but with the exception that there are still only two classes of an event and the specialisation considers only the method of how a check is performed.

At the end Layered Filtering follows the rule of thumb that the whole exceeds the sum of its parts.

Let's consider a sequence of layered anomaly detectors $L d_{1}, \ldots, L d_{n}$ where $n$ is the number of layers. Each detector has one input stream and two output streams: $a$-stream for anomalous and $n$-stream for normal events. A detector $L d_{i}$ passes to $L d_{i+1}$ only those events which are classified as anomalous. Among them there could be a lot of false positives but this is not important at this point. A Detector can perform any processing or transformations of events, under the condition that $a-$ stream of $L d_{i}$ is compatible with input stream of $L d_{i+1}$. The 


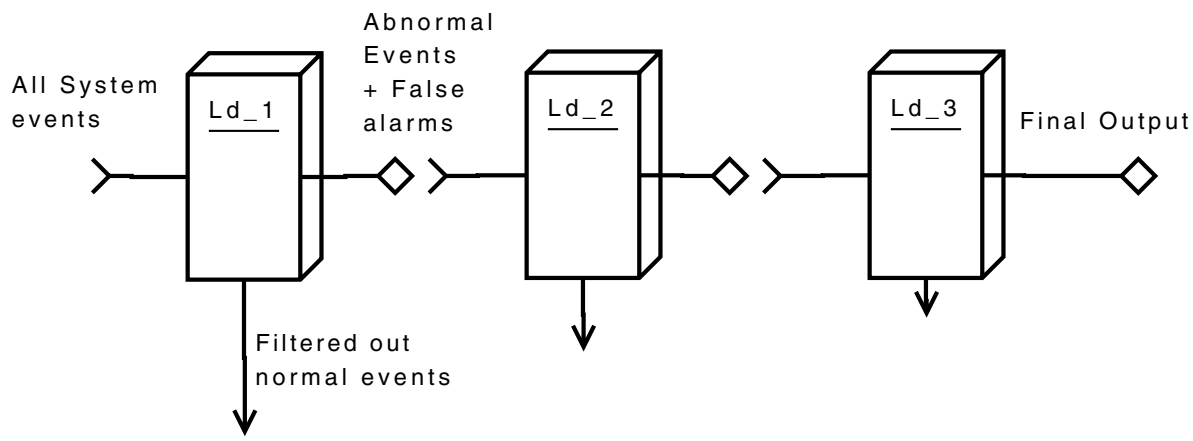

Fig. 1. Layered filtering schema

first and last detectors are distinguished in a sense that $L d_{1}$ must accept event types of system under supervision and $L d_{n}$ outputs information about anomalies to security officer. Figure 1 presents a schema for an anomaly detector based on layered filtering.

Let introduce the following symbols:

- $D T_{i}$ - i-th layer detection rate

- $F A R_{i}$ - i-th layer false alarm rate

- $P(I)_{i}$ - i-th layer base rate of anomalous events

- $P(\neg I)_{i}$ - i-th layer base rate of normal events

- $B D R_{i}$ - i-th layer bayesian detection rate

- $B T N R_{i}$ - i-th layer bayesian true negatives rate

Because of its operational characteristics the detector $L d_{i+1}$ operates on a set of events with significantly changed base rates of anomalies and non-anomalies - this is the most important part as it reduces the base-rate fallacy influence on a real false alarm rate. The base rates probabilities for the next layer are expressed in the following way:

$$
\begin{aligned}
P(I)_{i+1} & =B D R_{i} . \\
P(\neg I)_{i+1} & =1-B D R_{i} .
\end{aligned}
$$

It is now possible to write equations for $B D R_{i+1}$ and $B T N R_{i+1}$ as functions of, respectively $B D R_{i}$ and $B T N R_{i}$ :

$$
\begin{aligned}
B D R_{i+1} & =\frac{B D R_{i} * D R_{i+1}}{B D R_{i} * D R_{i+1}+\left(1-B D R_{i}\right) * F A R_{i+1}} . \\
B T N R_{i+1} & =\frac{\left(1-B D R_{i}\right) *\left(1-F A R_{i+1}\right)}{\left(1-B D R_{i}\right) *\left(1-F A R_{i+1}\right)+B D R_{i} *\left(1-D R_{i+1}\right)} .
\end{aligned}
$$

In this method requirements for operational characteristics for $L d_{i}$ can be relaxed significantly in terms of false alarm rate. However still it is best to have detection rate as high as possible. Additional advantage is that efficiency, 
in terms of system resources usage, should be improved because avoiding false alarms is one of the most complicated task for anomaly detector. What is more quantity of events that reaches further layers is greatly reduced and it makes it possible to use there more sophisticated algorithms without the risk of significant increase in the overall computational complexity.

\section{$5 \quad$ Results and Example IDS}

In this section results for two example layered filters are shown. In both cases the initial frequencies of normal and abnormal events are taken from previous section. These frequencies seen by next layers are calculated using (5) and (6). $B D R$ and $B T N R$ values are calculated using (7) and (8).

Table 1. Results for the first layered filter

\begin{tabular}{c|ccc}
\hline & $L d_{1}$ & $L d_{2}$ & $L d_{3}$ \\
\hline$P(I)_{i}$ & 0.00002 & 0.66667 & 0.99994 \\
$P(\neg I)_{i}$ & 0.99998 & 0.33333 & 0.00005 \\
$D R_{i}$ & 1.0 & 0.98 & 0.98 \\
$F A R_{i}$ & 0.00001 & 0.0001 & 0.00001 \\
\hline$B D R_{i}$ & 0.66667 & 0.99994 & 0.99999 \\
$B T N R_{i}$ & 1 & 0.96153 & 0.00254 \\
\hline
\end{tabular}

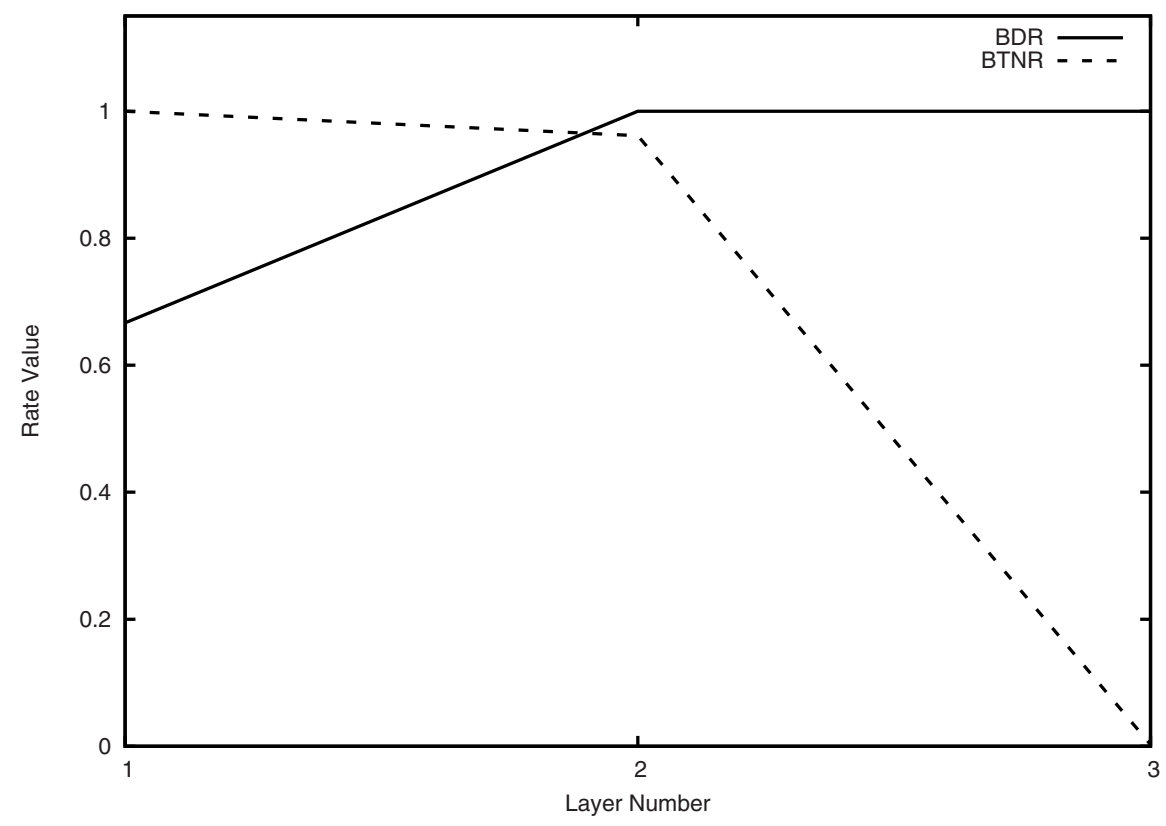

Fig. 2. $B T N R$ and $B D R$ changes for each layer of the first filter 
Table 2. Results for the second layered filter

\begin{tabular}{c|ccc}
\hline & $L d_{1}$ & $L d_{2}$ & $L d_{3}$ \\
\hline$P(I)_{i}$ & 0.00002 & 0.000391 & 0.27135 \\
$P(\neg I)_{i}$ & 0.99998 & 0.99960 & 0.72864 \\
$D R_{i}$ & 0.98 & 0.95 & 0.98 \\
$F A R_{i}$ & 0.05 & 0.001 & 0.0001 \\
\hline$B D R_{i}$ & 0.000391 & 0.271353 & 0.999726 \\
$B T N R_{i}$ & 0.999999 & 0.999980 & 0.99260 \\
\hline
\end{tabular}

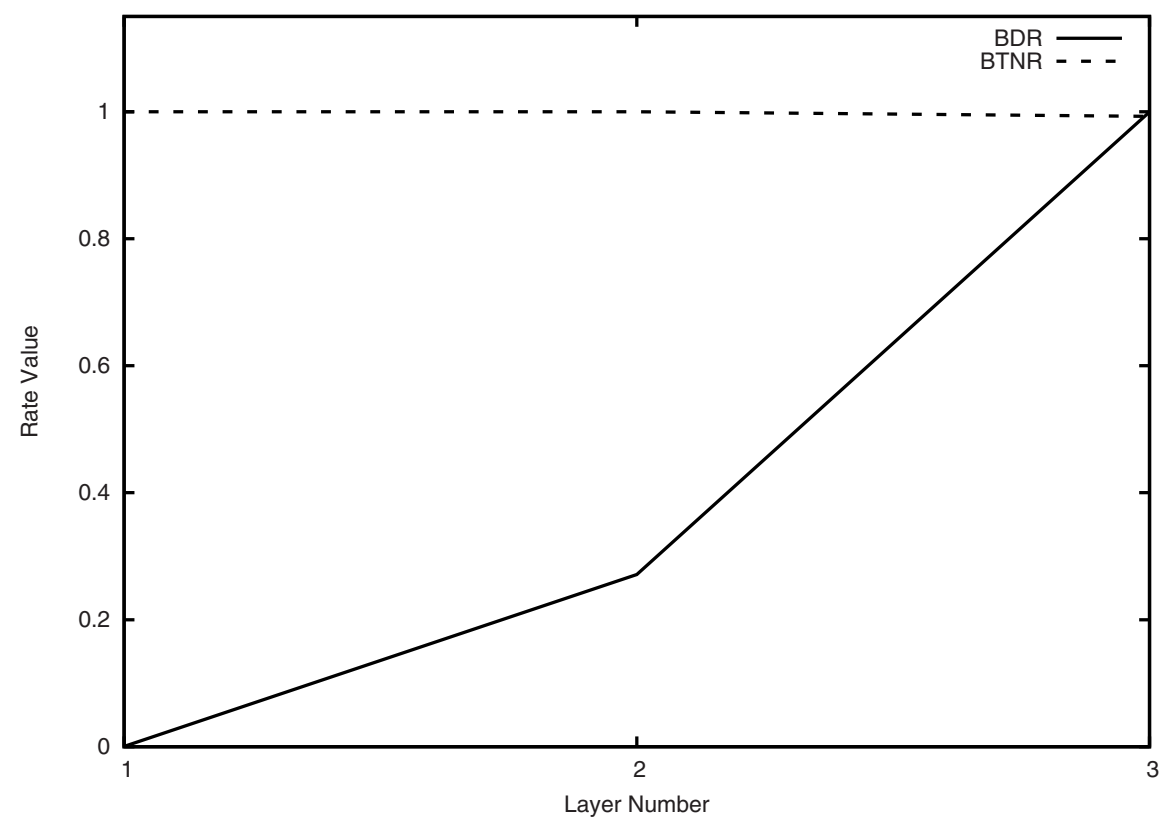

Fig. 3. $B T N R$ and $B D R$ changes for each layer of the second filter

First filter consists of three layers. Characteristics of each layer detector were based on assumptions from [2] and are rather unrealistic. Table 1 shows the calculated probabilities of normal and abnormal events, operational characteristics, $B D R$ and $B T N R$ values for each layer. It can be noticed that $B D R$ for the first filter increases significantly for the second layer and also $B T N R$ value falls dramatically for the third layer. In order the anomaly detector to be effective these both values must be maximised. As this is natural that increase of one of these values causes decrease of the second one the right balance must be found of layers quantity and detector characteristics. Figure 2 shows the plot of how the $B D R$ and $B T N R$ values changes for each layer. 
For the Second filter there have been a more realistic assumptions made, for the false alarm rates and detection rates of each layer, based on evaluations from 3. This filter also consists of three layers. In this case the increase of $B D R$ is a bit slower and also $B T N R$ stays on acceptable levels for all layers. Table 2 shows the calculated values. Figure 3 presents the relation of the layer number and $B D R(B T N R)$ values.

A form of real implementation of layered filter can be found in [1]. The system presented there is not directly referenced as layered filter but it consists of one layer based on Variable Order Markov Chain and the second one based on neural networks and multiagent systems. False alarm rate achieved there is actually 0 but the tests have been performed on a limited number of data sets and there have been a help from additional mechanism for suppressing false alarms called "anergic agents".

\section{Conclusions and Further Work}

This article shows that layered filtering approach for anomaly detection has a potential in reducing false alarm rate. Furthermore it can help in reducing computational complexity because of relaxed requirements especially for the first layer. However each layer detector must be carefully chosen in terms of performance and effectiveness. Also the balance between $B D R$ and $B T N R$ must be monitored as for certain number of layers $B T N R$ starts to fall very quickly. At the end it would be a mistake to use a detector based on the same methods in more then one layer as probably no additional classification decisions would be made.

The method presented here focuses on reducing false alarm rate but similar approach can be taken in reducing false negative rate by taking the second output from previous layer and connecting to it some sort of specialised detector which can verify if any anomaly has skipped detection and eventually redirect it to the next layer. It is also possible to build a two dimensional network of detectors for more sophisticated system for reducing both values.

Additional important remark can be done about constructing network security system based on signatures and anomaly detectors. Placing signature based detector on the first line of defence changes the base rates in the wrong way and makes anomaly detector useless. First line in detector stack must be held by system based on anomaly detection with proper characteristics minimising false negative rate. Second line may consists of signature based system or another anomaly detector. The work presented here may be an important input to the process of building network security from more then one intrusion detection system.

It is very interesting how operational characteristics change with number of layers. Further work includes implementation of IDS which is based on layered filtering and performing tests on real life environments. Also further research is needed on other methods of increasing intrusion detection systems effectiveness in terms of false negative and false positive rates. 


\section{References}

1. Cetnarowicz, K., Rojek, G., Pokrywka, R.: Intelligent Agents as Cells of Immunological Memory. In: Alexandrov, V.N., van Albada, G.D., Sloot, P.M.A., Dongarra, J. (eds.) ICCS 2006. LNCS, vol. 3993, pp. 855-862. Springer, Heidelberg (2006)

2. Axelsson, S.: The Base-Rate Fallacy and the Difficulty of Intrusion Detection. ACM Trans. Inf. Syst. Secur. 3, 186-205 (2000)

3. Warrender, C., Forrest, S., Perlmutter, B.: Detecting Intrusions Using System Calls: Alternative Data Models. In: IEEE Symposium on Security and Privacy, pp. 133145 (1999)

4. Klautau, A., Jevtic, N., Orlitsky, A.: Combined Binary Classifiers with Applications to Speech Recognition. In: International Conference on Spoken Language Processing 2002, pp. 2469-2472 (2002) 